\title{
Study of the performance of imidazolium-derived cations as structure directing agents in the synthesis of zeolites in fluoride media
}

\author{
Y. M. Variani, ${ }^{a}$ A. Rojas, ${ }^{\mathrm{a}, \mathrm{b}, *}$ L. Gómez-Hortigüela, ${ }^{\mathrm{c}}$ and S. B. C. Pergher ${ }^{\mathrm{a}}$
}

Three imidazolium cations based on the imidazolium ring, 1,2,3-triethylimidazolium (123TEI), 1,2,3-triethyl-4-methylimidazolium (123TE4MI), and 2-ethyl-1,3-dimethyl (2E13DMI), were employed as Structure Directing Agents (SDA) in the synthesis of pure silica zeolites, and led the crystallization of the STF zeolite. The three cations showed a different specificity for this phase, following the order 123TE4MI > 123TEI > 2E13DMI. Nevertheless, the 123TEI cation was also able to produce zeolite MFI when considerably high $\mathrm{H}_{2} \mathrm{O} / \mathrm{SiO}_{2}$ ratios were employed. In order to understand the selectivity of these imidazolium-based SDAs and explain the experimental results, a computational study was performed, showing a perfect agreement with the experimental observations. In the case of the MFI framework, the order of interaction energies found for this framework was 123TEI > 123TE4MI > 2E13DMI, providing an explanation to the experimental results. Hence, the $123 \mathrm{TEI}$ cation presents a certain template effect in terms of a spatial relationship between the shape of the organic cation and the topology of the MFI structure. A higher degree of motion of the 123TEl cation is observed when occluded within the cavities of the STF framework compared to when hosted in the channel intersections of the MFI framework, explaining the different widths of the ${ }^{13} \mathrm{C} N M R$ bands in the two materials.

\section{Introduction}

Zeolites are among the most intensively researched porous materials. The interest in these materials is not only related to their versatility, but also to a wide range of potential applications such as catalysts, ${ }^{1}$ adsorbents, ${ }^{2}$ chemical sensors, ${ }^{3}$ optical/electronic nanodevices ${ }^{4}$ and others. All these possible application fields are related to their great topological diversity, wide compositional range and high hydrothermal stability. In this sense, in the last years there has been an important advancement in the understanding of the factors that govern the zeolite crystallization pathways under different synthesis conditions (Structure Directing Effects), which is considered one of the principal research goals in the synthesis of this kind of materials. Thereby, it is possible to achieve a better control of the crystallization of a particular zeolitic phase by aiding in the stabilization of a less stable zeolite through strong host/guest interactions, or even to create new molecular sieve materials provided with new properties for advanced applications.

Different Structure Directing effects have been long-time recognized, such as i) the use of organic species as Structure Directing Agents (SDA), generally organic cations, that are designed with a particular molecular size and shape through an analysis of the conditions where the SDA has a higher geometric specificity for the particular structure that we want to crystallize, ${ }^{5}$ ii) the use of fluoride anions, which tend to favor structures with double-4 ring (D4R) units in the framework ${ }^{6}$ or iii) the isomorphic replacement of the tetrahedral framework atoms by other elements such as Al, B or Ge. ${ }^{7}$ Another fundamental variable that determines the output of a zeolite synthesis is the gel concentration, especially the $\mathrm{H}_{2} \mathrm{O} / \mathrm{SiO}_{2}$ ratio: the so-called Villaescusa's rule states that lower-density phases are favored at low $\mathrm{H}_{2} \mathrm{O} / \mathrm{SiO}_{2}$ ratios. ${ }^{6 \mathrm{c}}$ In addition, the crystallization temperature, time and regime (static or under agitation) can also influence on the final zeolitic phase that crystallizes.

Recently, organic cations based on the imidazolium ring, which is an aromatic rigid-cycle system with a high electronic density, have shown an efficient behavior as SDAs. Thus, previous reports have shown that the use of these SDAs together with optimized synthesis conditions enabled the synthesis of new zeolitic frameworks (CIT-7, ${ }^{8}$ $\left.\mathrm{IM}-16{ }^{9}{ }^{1 T W^{6 C}}\right)$, new compositions of zeolite structures already known ${ }^{10,11,12}$ or to achieve a greater stability of certain zeolitic phases. ${ }^{13}$ Taking into account these premises, in this work we have investigated the performance of three different organic cations based on the imidazolium ring (Figure 1) as SDAs in the synthesis of pure silica zeolites by the fluoride route. With this purpose in mind, 1,2,3-triethylimidazolium, 1,2,3-triethyl-4methylimidazolium and 2-ethyl-1,3-dimethylimidazolium cations were synthesized, characterized and their structure-directing ability for the synthesis of zeolite materials has been studied through a combination of experimental and computational methodologies. Important factors that may influence the performance of these molecules as SDAs towards different zeolite frameworks, such as their size, shape, conformational flexibility and hydrophobicity, have been investigated in the present work.

\section{Experimental section}

\section{Synthesis of the organic cations}

The organic SDAs that were used in the crystallization of zeolitic structures, a series of three different cations based on the imidazolium ring (see Figure 1), were synthesized by a double methylation or ethylation at the $\mathrm{N}$ position of the appropriately substituted primary imidazoles. 
Double Ethylation

1,2,3-triethylimidazolium (123TEI) and 1,2,3-triethyl-4-methylimidazolium (123TE4MI):

First $10 \mathrm{~g}$ of either 2-ethylimidazole or 2-ethyl-4-methylimidazole (Aldrich $99 \%$ ) was dissolved in $50 \mathrm{~mL}$ of chloroform (Aldrich 98\%). The obtained solution was mixed with an excess of iodoethane (1 pristine imidazole : 2 iodoethane molar ratio) in the presence of potassium carbonate sesquihydrate (Aldrich 99\%). After stirring for two days at room temperature, a second addition of iodoethane (in the same proportion) was carried out. The resulting mixture was kept under magnetic stirring for 4 days more. The inorganic phase was separated from the mother solution by filtration and the desired salt was obtained by rotatory evaporation under vacuum.

\section{Double Methylation:}

2-ethyl-1,3-dimethylimizolium:

For the synthesis of this cation, the same methodology as described above was employed, using 2-ethylimidazole (as pristine imidazole) and iodomethane (Aldrich 99\%) to carry out the methylation.

The yield of each cation synthesis was around $98 \%$ and the nature and purity of the products were confirmed by ${ }^{13} \mathrm{C}$ NMR (Figure S1 ESI + ).

The obtained iodide salts were then converted into the corresponding hydroxide forms by anion-exchange in aqueous solution using Dowex Monosphere 550A (OH) anion-exchange resin (Aldrich). After 24 hours under magnetic stirring at room temperature, the hydroxide solution was recovered by filtration and was concentrated by rotoevaporation under vacuum at $80^{\circ} \mathrm{C}$. The concentration of the hydroxide solution was monitored by titration with $\mathrm{HCl} 0.1 \mathrm{~mol} / \mathrm{L}$ using phenolphthalein as indicator before and after concentration.

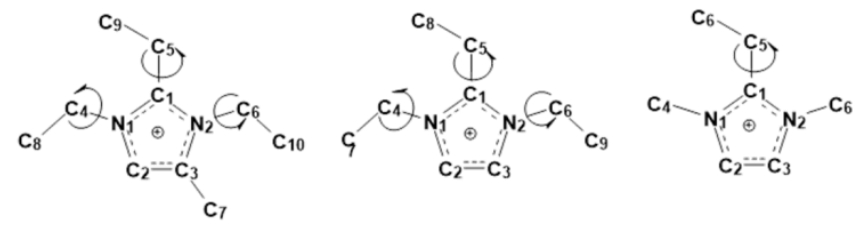

123TE4MI 2ETEI 2E13DMI

Figure. 1. Organic cations used in this work as SDAs. Each organic cation shows their rotatable bonds: three bonds for $123 \mathrm{TE} 4 \mathrm{MI}$ and 123TEI: $\mathrm{N}_{1}-\mathrm{C}_{4}-\mathrm{C}_{8(7)}, \mathrm{N}_{2}-\mathrm{C}_{6}-\mathrm{C}_{10(9)}, \mathrm{C}_{1}-\mathrm{C}_{5}-\mathrm{C}_{9(8)}$, and one for 2E13DMI: $\mathrm{C}_{1}-\mathrm{C}_{5}-\mathrm{C}_{6}$.

\section{Zeolite synthesis}

For the synthesis of zeolites, tetraethylorthosilicate (TEOS, Aldrich 98\%) was hydrolyzed at room temperature under stirring in an aqueous solution of the hydroxide form of the corresponding organic SDA. All the ethanol produced by the hydrolysis and water were allowed to evaporate and stirring was stopped when the desired composition was achieved. The amount of water was monitored by weight. At the end, HF (Cinética, 40\%) was added to the previous system under agitation with a spatula for 15 minutes. The final composition was $\mathrm{SiO}_{2}: 0.5 \mathrm{SDAOH}: 0.5 \mathrm{HF}: \mathrm{xH}_{2} \mathrm{O}$ and the $\mathrm{H}_{2} \mathrm{O} / \mathrm{SiO}_{2}$ ratio, ' $x$ ', was varied between 3.5 and 7.0. The obtained gels were distributed in Teflon lined stainless steel autoclaves which were heated at 160 or $175^{\circ} \mathrm{C}$ under static conditions. The autoclaves were taken out from the oven at different times and the solid products were isolated by filtration, washed with water and dried at $100^{\circ} \mathrm{C}$.

\section{Characterization}

The crystalline phases of the solids were identified by powder X-Ray Diffraction (XRD), recorded in a Bruker D2 Phaser diffractometer, using $\mathrm{Cu} \mathrm{K}_{\alpha}$ radiation between 5 to $50^{\circ} 2 \theta$ values. Scanning electron microscopy (SEM) was carried out with a HITACH TM3000 stand microscope with secondary electron emission. Multinuclear magic angle spinning (MAS) Nuclear Magnetic Resonance Spectra $\left({ }^{13} \mathrm{C},{ }^{29} \mathrm{Si}\right)$ were collected at room temperature on an Agilent Technologies DD2 equipment (details are given in the ESI ${ }^{\dagger}$ ). The amount of organic matter occluded in the zeolite was determined by CHN elemental chemical microanalysis on a LECO-CNNS 932 analyzer. The thermogravimetric analyses were performed on a Shimadzu, model DTG-60AH, where the samples were analyzed from RT $\left(25^{\circ} \mathrm{C}\right)$ to $1000^{\circ} \mathrm{C}$ with a heating rate of $10^{\circ} \mathrm{C} / \mathrm{min}$ and an air flow of $100 \mathrm{~mL} / \mathrm{min}$, using between 5 and $10 \mathrm{mg}$ of solid material for each analysis.

\section{Computational details}

The computational methodology employed to study the structure directing effect of the different imidazolium derivatives for the crystallization of the zeolite structures was based on molecular mechanics simulations, as implemented in Forcite module in Materials Studio. ${ }^{14}$ The geometry of the zeolite structures have been kept fixed during all the calculations. Molecular structures and the interaction energies of the organic SDAs with the framework are described with the CVFF forcefield; ${ }^{15}$ periodic boundary conditions (PBC) were applied in all the calculations. The atomic charge distributions of the organic imidazolium cations were obtained from DFT calculations, using the B3LYP hybrid functional and the ESP charge calculation method, setting the total net charge to +1 . The positive charge of the organic SDA molecules was compensated by the framework by using a related version of the uniform charge background method, ${ }^{16}$ where the atomic charge for every silicon framework atom was reduced from 0.6 until charge neutrality. Framework oxygen charges were kept fixed to -0.3 . Systems with 
single unit cells without symmetry (P1) were used for both MFI $\left(\mathrm{Si}_{96} \mathrm{O}_{192}\right)$ and $\operatorname{STF}\left(\mathrm{Si}_{32} \mathrm{O}_{64}\right)$ frameworks, since both primary unit cells were large enough as to avoid computational artefacts (both include at least one complete cavity/channel intersection where individual molecules site).

Due to the presence of rotatable bonds, and the importance of molecular flexibility in struture-direction, ${ }^{16}$ an initial conformational analysis was performed. The conformational space of the organic cations was scanned by means of the Conformers Module in Materials Studio, ${ }^{18}$ using a systematic grid scan search method and optimising the molecular structures for each set of dihedral angles.

The molecules were docked in the structures in the zeolite framework by Monte-Carlo simulations by using the Sorption Module in Material Studio. The final most stable location for the SDA molecules were then obtained by means of simulated annealing calculations, as implemented in the Forcite module. Interaction energies were calculated by subtracting the energy of the molecules in vacuo to the total energy of the system; all the energy values are given in $\mathrm{kcal} / \mathrm{mol}$ per unit cell (or per molecule for the conformational analysis).

\section{Results and discussion}

The synthesis results using the different imidazolium cations and various $\mathrm{H}_{2} \mathrm{O} / \mathrm{SiO}_{2}$ concentrations are summarized in Table 1. All the SDAs employed as Structure Directing Agents (SDAs) are able to produce the zeolite STF, but each one of them shows a different specificity for this zeolitic phase (see Figure S3 ESI + ). 123TE4MI imidazolium cation shows the strongest specificity for the STF zeolite, since this phase crystallizes in a broader range of $\mathrm{H}_{2} \mathrm{O} / \mathrm{SiO}_{2}$ ratios, from 3.5 to 5.5. In contrast, lower concentrations $\left(\mathrm{H}_{2} \mathrm{O} / \mathrm{SiO}_{2}=7.0\right)$ only lead to dense phases (tridymite-type); under this composition, STF is only obtained at a low synthesis temperature $\left(160^{\circ} \mathrm{C}\right)$ and an extremely long time $(30$ days). $123 \mathrm{TEI}$ and 2E13DMI also directed the crystallization of the STF framework, but in a narrower range of synthesis conditions, especially 2E13DMI. On the other hand, apart from the STF framework, only the 123TEI cation is able also to produce another zeolitic phase, MFI in this case, depending on the water/silica ratio. With this cation, the MFI phase is favored when the $\mathrm{H}_{2} \mathrm{O} / \mathrm{SiO}_{2}$ ratio is increased $\left(\mathrm{H}_{2} \mathrm{O} / \mathrm{SiO}_{2}\right.$ between 5.6 to 7.0), whilst the STF zeolite is obtained at lower $\mathrm{H}_{2} \mathrm{O} / \mathrm{SiO}_{2}$ ratios (around 3.5). These results are in accordance with the experimental observation known as Villaescusa's rule, ${ }^{6 c}$ which states that lower-density phases are favored at low water-silica ratios (in this case the STF zeolite has a lower framework density, FD, of $17.3 \mathrm{Si} / 1000 \AA^{3}$, compared to that of MFI of 17.9 $\mathrm{Si} / 1000 \AA^{3}$ ). Despite their framework densities being very similar to each other, a small difference seems to be enough to observe this specificity behavior. As evidenced in Table 1, STF is metastable and transforms in situ into $\mathrm{MFI}$, favoring the latter at long crystallization times (see results for $\mathrm{H}_{2} \mathrm{O} / \mathrm{SiO}_{2}$ of 5.7 at $175^{\circ} \mathrm{C}$ ). $2 \mathrm{E} 13 \mathrm{DMI}$ has the poorest performance as SDA, since STF is the only zeolite obtained and only pure at long crystallization times ( 39 days) under specific compositions. In addition, at low water/silica ratio, a mixture of STF and an unidentified phase crystallizes. The well-known MFI framework is composed by two interconnected 10MR channel systems along the [010] (straight channels) and [100] (sinusoidal channels); the STF framework is instead formed by independent globular-shaped cavities delimited by 10-ring windows.

Comparing the synthesis results based on the three different organic cations, they perform similarly with regard to the crystallization of the zeolite STF, with the 123TE4MI and 2E13DMI cations showing the strongest and poorest directing-role towards this framework, respectively. Thereby, the STF structure-directing role of the imidazolium cations follows the order: 123TE4MI > 123TEI > 2E13DMI. However, the result obtained with the 2E13DMI cation contrasts with that obtained recently by Schmidt et al. ${ }^{19}$ who obtained different zeolitic phases (MTW, ITW, STW+ITW mixture and STF but at long crystallization time) using the same cation, but under dynamic (constant stirring) synthesis conditions. On the other hand, only the 123TEl cation was able to direct the crystallization of the $\mathrm{MFI}$ framework, and hence the MFI-structure-directing role could be expressed as $123 \mathrm{TEI}>>123 \mathrm{TE} 4 \mathrm{MI} \approx 2 \mathrm{E} 13 \mathrm{DMI}$. The small differences observed in the specificity of the organic cations concerning the phase selectivity observed in this work can be related to different molecular aspects of these organic cations, such as size, shape, conformational flexibility and/or hydrophobicity, which can play an important role in the synthesis of zeolites. In this sense, in recent works the hydrophobicity of different imidazolium cations (following the procedure reported by Kubota et al. $)^{20}$ has been evaluated, showing an important influence of the molecular structure on the hydrophobicity of these cations, and therefore, in their behavior as SDA. ${ }^{13}$ Taking into account the importance of the hydrophobicity of the cations, this property was analyzed for the three imidazolium cations used in this work (Table 2). As can be observed in Table 2, the 2E13DMI cation displays the lowest hydrophobicity, since this cation does not show any transfer from water to chloroform. As expected, the 123TE4MI presents the highest percentage of transfer from water to chloroform, while the 123TEI cation showed an intermediate percentage of transfer. These results follow the $\mathrm{C} / \mathrm{N}$ ratio for the three molecules, 3.5, 4.5 and 5.0 for 2E13DMI, 123TEI and 123TE4MI, respectively, showing that higher $\mathrm{C} / \mathrm{N}$ ratios involve a higher hydrophobicity, and hence a higher transfer to the organic phase. Based on these results, we could have expected that the bulkiest cation, in our case the 123TE4MI, should display a stronger specificity towards more porous structures (such as MFI). Nevertheless, this does not occur, since only the STF zeolite is favored with this cation, and therefore another reason must exist that prevents the crystallization of the MFI framework with this bulkier cation. We should remark that although under our synthesis conditions neither of these cations is capable of directing structures with 4DR, recently Schmidt et al. ${ }^{19}$ reported that it is possible to obtain zeolites that contain this type of cavities by using other synthesis conditions. 
Table 1. Summary of Synthesis Results

\begin{tabular}{|c|c|c|c|c|}
\hline SDA & $\mathrm{H}_{2} \mathrm{O} / \mathrm{SiO}_{2}$ & Time /days & Phase & $\mathrm{T} /{ }^{\circ} \mathrm{C}$ \\
\hline \multirow{12}{*}{ 123TEI } & \multirow{3}{*}{3.5} & 8 & STF & \multirow{3}{*}{175} \\
\hline & & 11 & STF & \\
\hline & & 17 & STF & \\
\hline & \multirow{3}{*}{5.6} & 5 & Amorphous & \multirow{3}{*}{160} \\
\hline & & 11 & MFI & \\
\hline & & 19 & $\mathrm{MFI}$ & \\
\hline & \multirow{2}{*}{5.7} & 8 & $\mathrm{MFI}+\mathrm{STF}$ & \multirow{2}{*}{175} \\
\hline & & 17 & $\mathrm{MFI}(+\mathrm{STF})^{\mathrm{a}}$ & \\
\hline & \multirow{4}{*}{7.0} & 4 & $\mathrm{MFI}$ & \multirow{4}{*}{175} \\
\hline & & 9 & MFI & \\
\hline & & 10 & MFI & \\
\hline & & 12 & $\mathrm{MFI}$ & \\
\hline \multirow{9}{*}{ 2E13DMI } & \multirow{3}{*}{4.0} & 7 & Amorphous & \multirow{3}{*}{175} \\
\hline & & 10 & $\mathrm{STF}+\mathrm{U}^{\mathrm{b}}$ & \\
\hline & & 13 & $\mathrm{STF}+\mathrm{U}^{\mathrm{b}}$ & \\
\hline & \multirow{3}{*}{5.4} & 6 & Amorphous & \multirow{3}{*}{175} \\
\hline & & 12 & $\mathrm{STF}+\mathrm{U}^{\mathrm{a}}$ & \\
\hline & & 14 & $\mathrm{U}^{\mathrm{a}}$ & \\
\hline & \multirow{3}{*}{5.5} & 4 & Amorphous & \multirow{3}{*}{160} \\
\hline & & 11 & Amorphous+STF & \\
\hline & & 39 & STF & \\
\hline \multirow{12}{*}{ 123TE4MI } & \multirow{6}{*}{5.5} & 5 & STF & \multirow{3}{*}{175} \\
\hline & & 7 & STF & \\
\hline & & 10 & STF & \\
\hline & & 7 & STF & \multirow{3}{*}{175} \\
\hline & & 12 & STF & \\
\hline & & 16 & STF & \\
\hline & \multirow{3}{*}{7.0} & 7 & Amorphous & \multirow{3}{*}{175} \\
\hline & & 10 & Amorphous+Dense $^{c}$ & \\
\hline & & 17 & Amorphous+Dense $^{c}$ & \\
\hline & \multirow{3}{*}{7.0} & 9 & Amorphous & \multirow{3}{*}{160} \\
\hline & & 16 & Amorphous & \\
\hline & & 30 & STF & \\
\hline
\end{tabular}

${ }^{a}$ Minor presence

${ }^{\mathrm{b}}$ Unknown Phase

${ }^{\mathrm{c}}$ Tridymite-type dense phase

Table 2. Transfer of the cations in iodide form from water to chloroform solutions

\begin{tabular}{|c|c|c|c|}
\hline Cations & $(C+N) / q$ & $\mathrm{C} / \mathrm{N}$ & $\%$ Transfer \\
\hline $2 \mathrm{E} 13 \mathrm{DMI}^{+} \mathrm{I}^{-}$ & 9 & 3.5 & 0 \\
\hline 123TEI'I- & 11 & 4.5 & 3.2 \\
\hline 123TE4MI'I- & 12 & 5.0 & 8.2 \\
\hline
\end{tabular}

CHN chemical analysis of the as-made 123TEI-MFI zeolite (Table 3 ) shows $\mathrm{C} / \mathrm{N}$ ratios very close to the theoretical value of the SDA (4.5), suggesting that the imidazolium cations resisted the hydrothermal treatment and are incorporated intact within the framework. On the other hand, the STF zeolites obtained with 123TEI, 123TE4MI and 2E13DMI showed also $\mathrm{C} / \mathrm{N}$ ratios similar to the expected values, again suggesting the integral incorporation of the molecules. All these STF materials showed an incorporation of close to two imidazolium cations per unit cell $\left(\left[\mathrm{SiO}_{2}\right]_{32}\right)$, resulting in an incorporation of one imidazolium cation per STF cavity.

Figure $\mathrm{S} 2$ shows the thermogravimetric analysis (TG/DTG) of the materials obtained with the three different cations. The 123TEI-MFI material shows two principal weight losses, which are related to the decomposition of the organic

Table 3. $\mathrm{CHN}$ chemical analyses of the most interesting samples obtained.

\begin{tabular}{|c|c|c|c|c|c|c|c|}
\hline Phase & $\% \mathrm{C}$ & $\% \mathrm{H}$ & $\% \mathrm{~N}$ & $\mathrm{C} / \mathrm{N}^{\mathrm{b}}$ & $\mathrm{H} / \mathrm{N}^{\mathrm{b}}$ & TG & Formula $^{c}$ \\
\hline 123TEI-MFI & 8.2 & 1.2 & 2.0 & $4.7(4.5)$ & $8.5(8.5)$ & 88.2 & $\left|\mathrm{C}_{9} \mathrm{~N}_{2} \mathrm{H}_{17} \mathrm{~F}\right|_{4.1}\left[\mathrm{SiO}_{2}\right]_{96}: 0.3 \mathrm{H}_{2} \mathrm{O}$ \\
\hline 123TEI-STF & 9.1 & 1.6 & 2.5 & $4.4(4.5)$ & $8.9(8.5)$ & 84.7 & $\left|\mathrm{C}_{9} \mathrm{~N}_{2} \mathrm{H}_{17} \mathrm{~F}\right|_{1.9}\left[\mathrm{SiO}_{2}\right]_{32}: 0.7 \mathrm{H}_{2} \mathrm{O}$ \\
\hline 123TE4MI-STF & 9.8 & 1.5 & 2.1 & $5.4(5.0)$ & $10.0(9.5)$ & 83.7 & $\left|\mathrm{C}_{10} \mathrm{~N}_{2} \mathrm{H}_{19} \mathrm{~F}\right|_{1,8}\left[\mathrm{SiO}_{2}\right]_{32}: 0.9 \mathrm{H}_{2} \mathrm{O}$ \\
\hline 2E13DMI-STF ${ }^{a}$ & 7.7 & 1.1 & 2.4 & $3.7(3.5)$ & $6.5(6.5)$ & 91.3 & $\left|\mathrm{C}_{7} \mathrm{~N}_{2} \mathrm{H}_{13} \mathrm{~F}\right|_{1.9}\left[\mathrm{SiO}_{2}\right]_{32}: 0.02 \mathrm{H}_{2} \mathrm{O}$ \\
\hline
\end{tabular}

${ }^{\mathrm{a}}$ Synthesis at $160^{\circ} \mathrm{C}$. ${ }^{\mathrm{b}}$ Molar ratio. The theoretical value is given in parentheses. ${ }^{\mathrm{c}} \mathrm{SDA}$ from $\mathrm{N}$ analysis assuming the SDA is intact. The SDAs positive charges are assumed to be balance only by $\mathrm{F}$ anions. Water derived from the excess $\mathrm{H} / \mathrm{N}$ ratio (it may represent both water content and/or SiOH defects). 
cation at $418^{\circ} \mathrm{C}$ and $511^{\circ} \mathrm{C}$. In the case of the STF obtained with the $2 \mathrm{E} 13 \mathrm{DMI}$ cation, only one main weight loss related to the SDA elimination at $450^{\circ} \mathrm{C}$ is appreciated, while the $123 \mathrm{TEI}$-STF and 123TE4MI-STF materials show similar profiles, with two consecutive steps for the elimination of the SDA. The first one is likely due to the decomposition of the cation occluded inside the STF structure, occurring at around $405^{\circ} \mathrm{C}$, followed by the elimination of the residue produced by an incomplete combustion around $520^{\circ} \mathrm{C}$, which is evidenced in the TG curve by a weight loss of $7 \%$ in both cases.

The ${ }^{13}$ C MAS NMR spectra of the materials obtained with the three different SDAs are shown in Figure 2. All the STF zeolites synthesized with the different imidazolium cations showed NMR spectra with sharp and intense bands which could be assigned to the different $C$ atoms of the imidazolium cations, indicating that the SDAs are intact inside the zeolite frameworks. The MFI and STF spectra obtained with the 123TEl cation show different signals at low chemical shifts (5-20 ppm) corresponding to the ethyl groups, while the resonance associated to the carbons bonded to the $\mathrm{N}$ and imidazolium ring appear around $120 \mathrm{ppm}$ and $130 \mathrm{ppm}$, respectively. Nonetheless, in the case of 123TEI-MFI, the bands are much broader, and are not well resolved. However, the resonances are found in the right chemical shift ranges (although the bands are slightly shifted for the 123TEl cations in the two different frameworks), and therefore, we can conclude that the 123TEl cation is mostly intact, in accordance with the CHN results. It is interesting to remark the notable differences in the band widths of the 123TEl cation occluded in the two frameworks: as previously mentioned, these bands are much sharper and well resolved in the STF structure. This observation could be explained either by the occurrence of different locations of the 123TEI cations in the MFI framework (what would broaden the NMR signals), or by a higher degree of motion of the cations located in the cavities of the STF framework (what would sharpen the NMR signals) compared to when located in the intersections of the MFI framework; this will be discussed below, with the aid of our computational simulations. The 2E13DMISTF spectrum shows two resonances between 120 to $150 \mathrm{ppm}$, corresponding to the three aromatic carbons, plus three additional signals in the 5-35 ppm region belonging to the methyl carbons bonded to $\mathrm{N}$ and the ethyl group. In the case of the 123TE4MI-STF spectrum, apart from the three aromatic carbons in the 115-145 ppm region, two (40 to $45 \mathrm{ppm}$ ) and five (5-20 ppm) resonances are observed, corresponding to the $\mathrm{C}-\mathrm{N}$ and the carbons belonging to the ethyl and methyl groups, respectively.

The ${ }^{29} \mathrm{Si}$ MAS NMR spectra of the MFI and STF as-made materials are shown in Figure 3. The 2E13DMI-STF (Fig. 3a) spectrum shows at least 5 crystallographic sites, between -104 and $-121 \mathrm{ppm}$, corresponding to $\mathrm{Q}^{4} \mathrm{Si}$ species $\left(\mathrm{Si}[\mathrm{OSi}]_{4}\right)$.

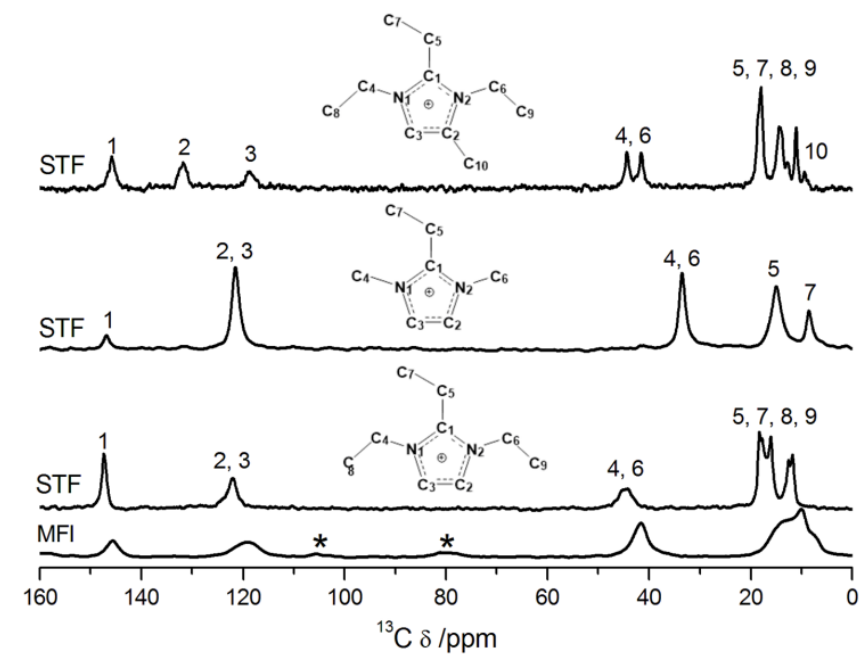

Figure 2. ${ }^{13} \mathrm{C}$ MAS NMR spectra of the as-made solids obtained with the three SDAs (from bottom to top): 123TEI-MFI, 123TEI-STF, 2E13DMI-STF, 123TE4MI-STF. Some impurities are marked with $\left({ }^{*}\right)$.

The 123TEI-STF material (Fig. 3b) exhibits also at least 5 different signals ( -105 to $-120 \mathrm{ppm}$ ), all of them corresponding to $Q^{4}$ species, although the chemical shifts and relative intensities differ from the previous case. In contrast, in the 123TE4MI-STF spectrum (Fig. 3c), at least 3 crystallographic sites (-104 to $-119 \mathrm{ppm}$ ) related to $Q^{4}$ species are observed, but the band at around $-104 \mathrm{ppm}$ observed in the previous samples is not clearly appreciated in this case. Beside, a small concentration of $\mathrm{Q}^{3}$ spices, i.e., connectivity defects $\left(\mathrm{Si}\left[(\mathrm{OSi})_{3} \mathrm{OH}\right]\right.$ or $\mathrm{Si}\left[(\mathrm{OSi})_{3} \mathrm{O}\right]^{-}$ evironments) are appreciated between -99 to $-102 \mathrm{ppm}$ in the spectra of the STF, especially in the one obtained with 123TE4MI (Figure 3c). On the other hand, there is evidence in the 123TEI-STF material of pentacoordinate units $^{21}\left[\mathrm{SiO}_{4 / 2} \mathrm{~F}\right]$ showing a resonance around $-145 \mathrm{ppm}$ (as was described for analogous materials), ${ }^{22,{ }^{23}}$ which can also be appreciated in the 2E13DMI-STF material; nevertheless apparently this resonance is not observed in the 123TE4MI-STF. There is an additional signal in this sample at $-92 \mathrm{ppm}$ whose origin is not clear at present. These results seem to indicate that the STF frameworks obtained with 2E13DMI and 123TEI contain fluoride anions (bonded to $\mathrm{Si}$ ) as charge-compensating defects to balance the positive charge of the organic cations, whilst in the 
STF framework obtained with the larger cation 123TE4MI, connectivity defects would provide charge-compensation (probably associated to Si vacancies leading to Si nests). Nevertheless, the reason for this different behavior is not clear at the moment.

Regarding the MFI framework, the 123TEI-MFI spectrum (Fig. 3d) shows a resonance with a high intensity in the range between -106 and $-124 \mathrm{ppm}$ associated to $\mathrm{Si} \mathrm{Q}^{4}$. Additionally, this spectrum shows another broad band between -97 to $-104 \mathrm{ppm}$, indicating again the presence of connectivity defects ( $Q^{3}$ units).
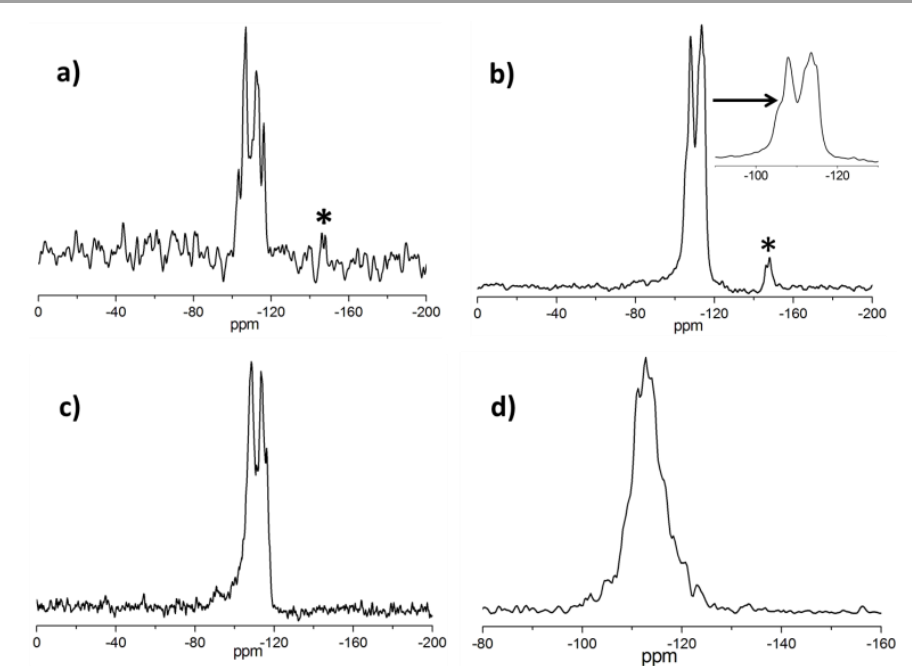

Figure 3. ${ }^{29}$ Si MAS NMR spectra of the as-made solids obtained with the three SDAs. a) 2E13DMI-STF, b) 123TEI-STF, c) 123TE4MISTF, d) 123TEI-MFI.

FE-SEM images (Figure S4 ESI + ) show that the 123TEI-MFI material crystallized in the form of long and thick prismatic star-shaped crystalline agglomerates, with a high aspect ratio. In the case of the STF zeolite obtained with the 123TEI and 123TE4MI cations, both showed similar morphologies with well-defined crystals. In contrast, the 2E13DMI-STF sample displayed interpenetrated plate-shaped crystals of different sizes.

\section{Computational results}

Our experimental results clearly showed that the tree closely-related imidazolium cations were able to drive the crystallization of the cavity-based STF framework, whilst only the 123TEI cation gave place to the 2D-channel system of the MFI framework. We then applied molecular-mechanics simulations in order to understand, from a molecular point of view, these different structure-directing behaviors by finding and comparing the location and interaction energies of the three cations in the two frameworks.

\section{A) Conformational analysis of the SDA cations}

Due to the importance of flexibility in structure-direction in molecules which possess single rotatable bonds, with the consequent occurrence of different conformers that could potentially be occluded within a zeolite framework, we initially analyzed the conformational space of the different cations. 2E13DMI, 123TEI and 123TE4MI cations have 1,3 and 3 rotatable bonds, respectively, that produce significantly different conformers (see Figure 4).

The conformational search around the rotatable $\mathrm{C}$ (ring)-C(ethyl) bond of 2E13DMI (this bond is the only one that leads to different molecular shapes) showed the presence of a unique stable conformer (after geometry optimization) to which all the conformers with different torsion angles reverted (Figure 4-top-left). Rotation around such bond must be slightly impeded because of the presence of the methyl substituents that provokes a steric hyndrance when the methyl and ethyl substituents are aligned with each other, although the conformational barrier might be low. 

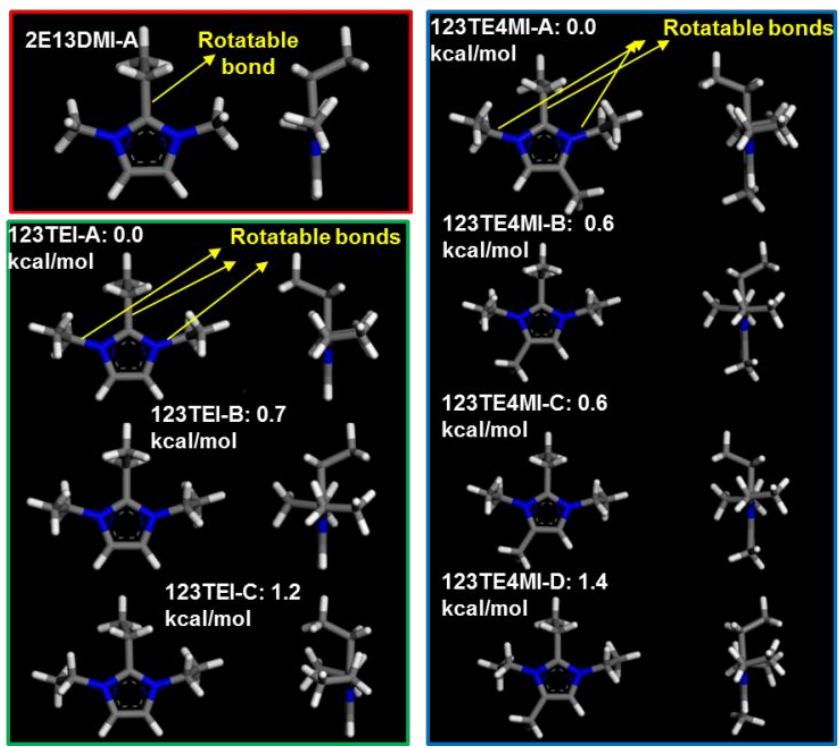

Figure 4. Most stable conformations (with relative energies lower than $2.0 \mathrm{kcal} / \mathrm{mol}$ with respect to the most stable conformer) of the cations employed in this work.

123TEI has three rotatable bonds that lead to different conformations: the conformational search (followed by geometry optimisation) led to 5 non-equivalent conformers (Figure 4-left, 123TEl, only the three most stable conformers are shown), depending on the relative orientation of the ethyl groups. The most stable one corresponds to a conformation where the two ethyl groups bonded to $\mathrm{N}$ point to the same side, and opposite to the central ethyl group (conformer A), avoiding any steric hindrance between the ethyl substituents. There is a small energy difference with conformers where the two ethyl groups attached to $\mathrm{N}$ are on opposite sides (conformer $\mathrm{B}, 0.7$ $\mathrm{kcal} / \mathrm{mol}$ ) or where the three ethyl groups are on the same side (conformer $\mathrm{C}, 1.2 \mathrm{kcal} / \mathrm{mol}$ ). The other two conformers, which have one of the ethyl groups attached to $\mathrm{N}$ coplanar with the imidazolium ring (conformers $\mathrm{C}$ and $\mathrm{D}$ ), are much less stable ( 2.9 and $3.4 \mathrm{kcal} / \mathrm{mol}$, respectively).

The most stable conformer of $123 \mathrm{TE} 4 \mathrm{MI}$ is similar to that of $123 \mathrm{TEI}$, with the two ethyl chains bonded to $\mathrm{N}$ pointing to the same side, and opposite to that of the central ethyl group (Figure 4-right). The other stable conformers are also similar to those of $123 \mathrm{TEI}$, differing in the relative orientation of the ethyl groups. In this case, the breakage of the symmetry because of the additional methyl group involves the existence of another non-equivalent conformer (C).

B) Structure-directing effect towards the MFI framework.

We first studied the incorporation of 2E13DMI molecules within the MFI framework. Monte-Carlo simulations showed that the molecules always located in the channel intersections, with 4 molecules per unit cell (one per channel intersection). The most stable location of the molecules was then obtained by simulated annealing calculations (Figure 5). Results showed that 2E13DMI molecules locate in the channel intersections, with the imidazolium ring in the intersection, and the ethyl groups in the sinusoidal channels. The location of the 2E13DMI molecules is highly ordered, and indeed the space group of the MFI framework (including the 2E13DMI cations) is Pnma (the same as that of the organic-free framework), showing that the arrangement of the organics follows the symmetry of the MFI framework (Pnma). The total interaction energy of the 2E13DMI cations (4 molecules per unit cell) is $-308.6 \mathrm{kcal} / \mathrm{mol}$ per u.c. (Table 4).

Table 4. Host-guest systems studied by molecular simulations.

\begin{tabular}{|c|c|c|c|c|c|}
\hline Framework & Molecule & Configuration & Molecular Conformation & Interaction E (kcal/mol per u.c.) & Space group \\
\hline \multirow{5}{*}{ MFI } & 2E13DMI & - & $\mathrm{A}$ & -308.6 & Pnma \\
\hline & \multirow{2}{*}{ 123TEI } & 1 & $A$ & -319.5 & $P m n 2_{1}$ \\
\hline & & 2 & $A$ & -318.8 & $P m n 2_{1}$ \\
\hline & \multirow{2}{*}{ 123TE4MI } & 1 & $A$ & -240.9 & $P 1$ \\
\hline & & 2 & Unstable & -311.2 & $P 2_{1} 2_{1} 2_{1}$ \\
\hline \multirow{5}{*}{ STF } & 2E13DMI & - & $A$ & -144.5 & $P 2_{1}$ \\
\hline & \multirow{2}{*}{ 123TEI } & 1 & $\mathrm{D}$ & -152.9 & $P 1$ \\
\hline & & 2 & $A$ & -151.5 & P1 \\
\hline & \multirow{2}{*}{ 123TE4MI } & 1 & $\mathrm{D}$ & -154.0 & $P 1$ \\
\hline & & 2 & $A$ & -154.7 & P1 \\
\hline
\end{tabular}


We then studied the docking of the 123TEI molecules within the MFI framework. In this case, the five different conformers were tried, by docking one molecule through the MC simulations, after which a simulated annealing procedure was carried out. Regardless of the conformer studied, all the simulations led to the same location (in the channel intersections) and with the cation in the same conformation (conformer A in Figure 4). A similar location to that of 2E13DMI is found, where the central ethyl group locates on the sinusoidal channels, and the imidazolium ring sites on the straight channels, in the intersection between both channel systems. In this case, the ethyl groups attached to $\mathrm{N}$ are roughly aligned with the straight channels. The adopted conformation is close to the most stable one (conformer A, Figure 4), although the ethyl groups bonded to $\mathrm{N}$ are slightly bent. The most stable location of the molecules ( 4 per unit cell, after simulated annealing) is shown in Figure 5 (middle); the total interaction energy calculated was $-319.5 \mathrm{kcal} / \mathrm{mol}$ per MFI u.c. (Table 4). The molecules again locate in a well-ordered arrangement, with the central ethyl groups (attached to the $\mathrm{C}$ atoms) in the sinusoidal channels (pointing towards opposite sides, configuration 1 in Table 4), and the two ethyl groups attached to the $\mathrm{N}$ atoms aligned with the straight channels. Indeed, the MFI framework with the molecules loaded still has a $P m n 2_{1}$ space group, revealing the high ordering of the organic molecules, which again follows the MFI framework symmetry. We tried with similar arrangements but where the molecules in the sinusoidal channels are pointing towards the same side (configuration 2, see Figure S5 ESIt) (not to opposite sides, as in Figure 4-middle), and similar energies $(-318.8 \mathrm{kcal} / \mathrm{mol}$ per u.c., compared to $319.5 \mathrm{kcal} / \mathrm{mol}$ for $123 \mathrm{TEl}$ pointing to opposite sides in configuration 1$)$ and symmetry $\left(P m n 2_{1}\right)$ were found.
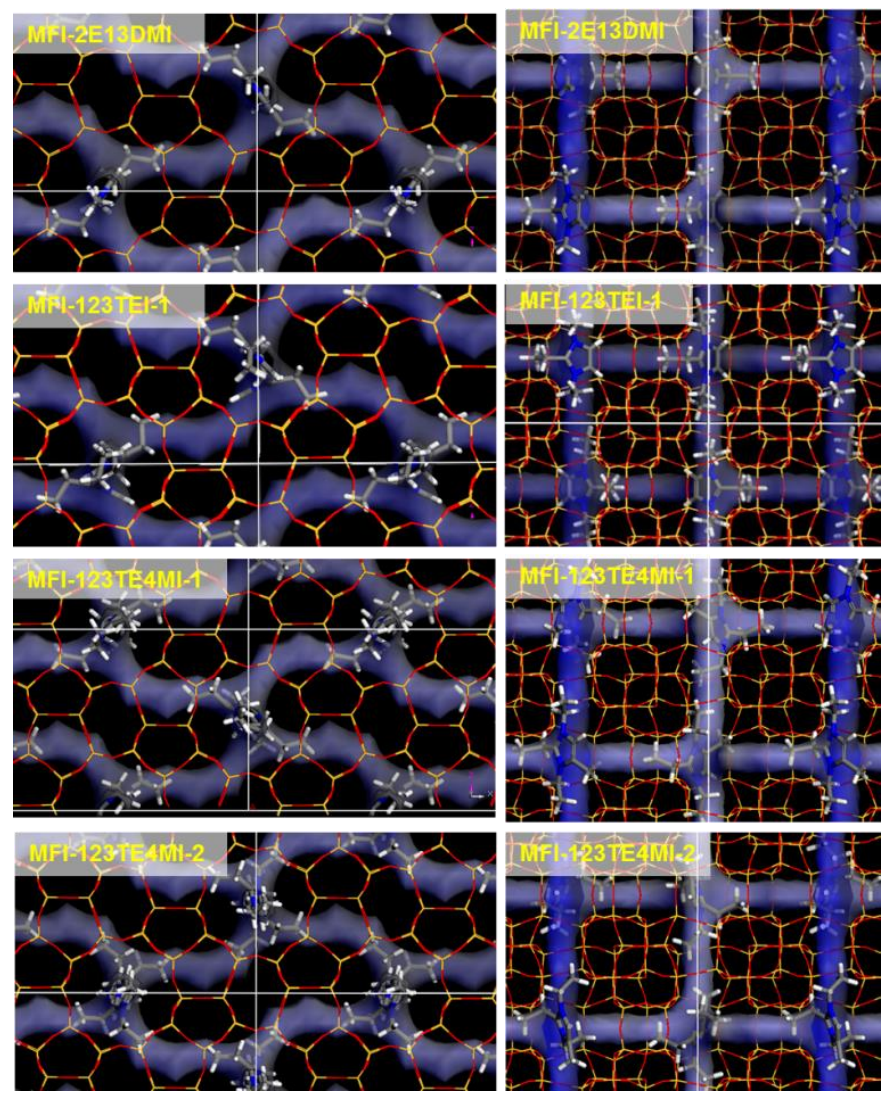

Figure 5. Location of the different cations within the MFI framework (with 4 molecules per unit cell).

The similar energies of both systems suggest that both configurations could actually occur during the actual crystallization of the framework.

We finally studied the incorporation of the 123TE4MI cations within the MFI framework. In this case, location of the molecules in the same site (in the channel intersections) and conformation (A) as those previously found for 2E13DMI and 123TEI molecules led to a dramatic decrease of the interaction energy $(-240.9 \mathrm{kcal} / \mathrm{mol}$, configuration 1 in Table 4). This is caused by a steric repulsion provoked by the methyl groups with the 10-MR channel walls (see dashed yellow circles in Figure 4-bottom, MFI-123TE4MI-1), indicating that the highly-ordered arrangement of the organic cations within the MFI framework found for 123TEI is not stable for 123TE4MI. Indeed, the conformation of the $123 \mathrm{TE} 4 \mathrm{MI}$ molecules in this configuration has an energy penalty of $12 \mathrm{kcal} / \mathrm{mol}$ per molecule, suggesting a high molecular constraint under this configuration. Simulated annealing calculations led to another configuration for the 123TE4MI molecules (Figure 4-bottom, MFI-123TE4MI-2), with an interaction energy of $-311.2 \mathrm{kcal} / \mathrm{mol}$ (configuration 2 in Table 4) (in this case the conformational energy penalty is lower, $6.2 \mathrm{kcal} / \mathrm{mol}$ ). This is clearly more stable than the previous configuration due to a better adjustment of the molecular conformation, which in this case sites two ethyl groups towards the sinusoidal channels, and the other ethyl and the methyl groups roughly 
aligned with the straight channels. However, still the interaction energy is notably lower than for 123TEl. The resulting symmetry in this case is $P 2_{1} 2_{1} 2_{1}$, suggesting that the molecules cannot follow the framework symmetry.

C) Structure-directing effect towards the STF framework.

We then analyzed the location of the molecules within the cavities of the STF framework (one molecule per cavity, equivalent to 2 per unit cell). In this case, the STF cavities will be filled by one single molecule, and therefore no lateral intermolecular packing interactions will be developed. Molecules were initially docked by MC simulations, and simulated annealing then led to the most stable configuration; again all the possible conformers were initially employed, but all of them reverted to the cases explained below. The interaction energies of the different systems (two different stable configurations were found for 123TEI and 123TE4MI molecules) and the final location of the molecules are shown in Table 4 and Figure 6.

2E13DMI molecules locate comfortably within the cavities, developing an interaction energy of $-144.5 \mathrm{kcal} / \mathrm{mol}$ per STF unit cell (see Figure 6-top), although they do not completely fill the STF cavities. Two different configurations for the 123TEI molecules are stable when occluded within the cavities of the STF framework (Figure 6), which correspond to conformer D (see Figure 4, with all the ethyl substituents at the same side) or conformer $A$ (the most stable one in vacuum), with similar stabilities $(-152.9$ and $-151.5 \mathrm{kcal} / \mathrm{mol}$, respectively, Table 4$)$. The same type of configurations are observed for 123TE4MI molecules (Figure 6), again with very similar stabilities for both cases (154.0 and $-154.7 \mathrm{kcal} / \mathrm{mol}$, respectively, Table 4). In the case of the STF host-guest systems, no clear geometrical or symmetry relationship between the molecules and the framework has been observed. The spherically-shaped imidazolium cations are just occluded within the spherical cavities of the STF framework, but no strong spatiallydirected interactions are established as in the case of MFI.

\section{Discussion}

The computational results match nicely and explain our experimental observations regarding the structure-directing behavior of the molecules towards the two framework types. Under the synthesis conditions of our host-guest set of systems, the STF framework seems to crystallize as the default structure, in the sense that a true template effect is not required. This is evidenced by the lack of any geometrical and/or symmetry relationship between the framework and the location of the molecules, and the fact that the three molecules are able to direct its crystallization. The STF framework is formed by spherical cavities which can comfortably accommodate the globular shape of the cations employed in this work. Indeed, different conformations can be accommodated within the cavities. The experimentally observed structure-directing efficiency follows the order 123TE4MI >123TEI > 2E13DMI , which is in perfect agreement with the order of interaction energies found 

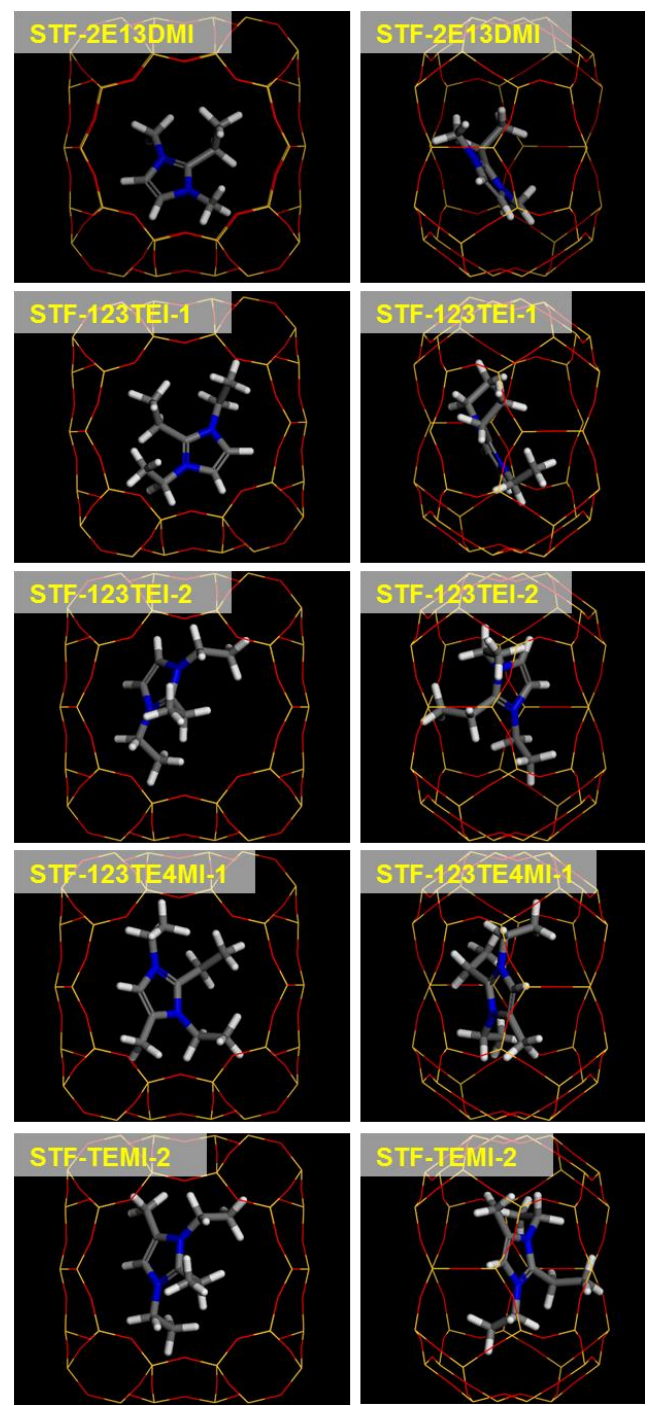

Figure 6. Location of the different cations within the STF framework (with 2 molecules per unit cell, one per cavity).

in our computational study, providing an explanation for the synthesis results. In this case, the structure-directing efficiency seems to be related just to the number of interacting guest $(\mathrm{C}+\mathrm{N})$ atoms able to be accommodated in the cavity, which will enhance the interaction with the zeolite host framework, while preventing steric hindrances. Hence the bulkiest molecule able to comfortably fit the cavity dimensions (123TE4MI) displays the strongest directing-ability towards the STF framework.

A different situation is observed for the crystallization of the MFI framework. In this case, the experimental observations indicate that only 123TEl is able to direct the crystallization of this framework, and therefore a true structure-directing effect is observed. Indeed, a certain template effect, in terms of the spatial relationship between the shape of the organic 123TEl cation and that of the MFI channel topology, has been found. The cation sites on a very specific position within the MFI framework, on the intersection between the two channel systems, with each ethyl group pointing towards one direction: the central ethyl chain pointing to the sinusoidal channels, and the other two aligned with the straight channels; thus, the ethyl groups allow for a good fitting of the imidazolium cation within the channels. Indeed, the template effect of this cation is evidenced also by the arrangement of the molecules that follows the symmetry of the MFI framework. In an attempt to provide an experimental confirmation of the highly-ordered arrangement of 123TEl cations within the MFI framework (taking into account the difficulty of finding the location of the cations by diffraction techniques due to the complexity of the MFI framework), we compared the XRD patterns of the as-made and calcined samples, and with the calculated pattern of simulated 123TEI-MFI model (see Figure S6 ESI ${ }^{\dagger}$ ). The ordered arrangement of the organics inside MFI causes a change in the symmetry that can be confirmed by the calculated XRD pattern of the simulated model, which agrees with the experimental pattern of the as-made material and shows reflections that are absent or are less intense in the calcined material (indicated by asterisks). This suggests that the arrangement found in the simulation actually occurs in the material.

The order of interaction energies found for this MFI framework, 123TEI > 123TE4MI > 2E13DMI, is also in very good agreement with the experimental observations. 2E13DMI molecules develop a lower interaction with the framework due to the lower number of atoms. Our simulations show that the filling of the sinusoidal channels by 
123TEI and 2E13DMI is essentially the same since these are filled by the central ethyl groups which are present in both molecules. The difference is on the alkyl groups attached to the $\mathrm{N}$ atoms, which locate aligned with the straight channels. These channels are much more efficiently filled by the 123TEI cations due to their larger size which nicely fits with the distance spanning consecutive channel intersections. In contrast, a poor-filling of the straight channels is achieved by the smaller methyl groups of the 2E13DMI cation, explaining the inability of this molecule to direct the MFI framework.

A different picture is observed for 123TE4MI cations. In this case, the arrangement of the molecules in the channel intersection as the one found for 123TEl, which showed a very good structure-directing
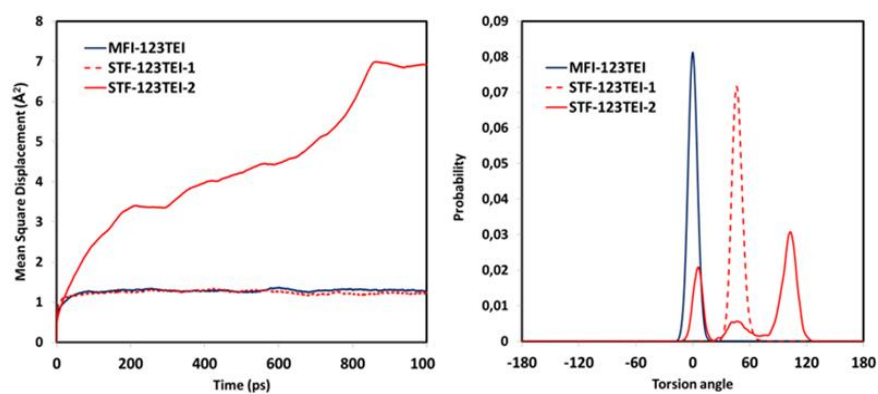

Figure 7. Mean Square Displacement (MSD, left) and torsion angle distribution between two molecular atoms and two framework atoms (right) for the different systems, both indicators of the molecular motion of the 123TEl cations in the different frameworks.

and templating effect, involves a dramatic decrease of the stability of the system, caused by the steric repulsion generated by the additional methyl group with the zeolite walls. Therefore, the presence of the methyl group prevents the location of the molecules in the highly-ordered arrangement in the MFI channel intersections that promote the crystallization of the framework. Another more stable location for the molecules has been found, although in this case there is not a clear geometrical and symmetry relationship between the molecule and the framework, and besides the interaction energy is notably lower, thus explaining also the inability of this molecule to promote the formation of the MFI framework.

Our results show then a different structure-directing behavior of the 123TEl cation towards the MFI or STF frameworks. In the first case, the molecule locates in a very specific position on the channel intersections, showing a good fitting with the geometry of the channel system; as a consequence, motion of this cation should be strongly restricted. We performed Molecular Dynamics simulations (at $298 \mathrm{~K}$ ) in order to analyze the actual motion of this cation within the MFI framework, and we observed no rotation or translation along the MD simulation; this was also confirmed by calculating the Mean Square Displacement (MSD), which is an indication of the motion of the molecule, that was very low (Figure 7-left, blue line). Besides, we calculated the torsion angle between two atoms of the cation and two atoms of the framework, and observed a very narrow and intense distribution, which is indicative of the lack of any rotation of the occluded molecule (Figure 7-right, blue line). Such lack of motion of the cation in the MFI framework could provide an explanation for the broad bands observed in the ${ }^{13} \mathrm{C}$ NMR spectrum (Figure 2), typical of solid-state NMR. In the case of the 123TEI cation directing the STF framework, there is not a specific position neither a relationship between the geometry of the framework and the molecular shape (beyond the fact that both the STF cavities and the cation have globular shapes), and in this case the structure-directing effect just consists on a filling of the STF cavities by the molecules. In this case, we found two stable locations for the molecules, with two different conformations. The MD simulations showed that in configuration 1, where the molecules had all the ethyl groups at the same side, conformation which is less stable in vacuum, no motion, either translation (see MSD in Figure 7-left, red-dashed line) or rotation (see torsion angle distribution in Figure 7-right, red-dashed line), was observed, suggesting that the molecules under this conformation are tightly confined. In contrast, a much higher translation (given by the high MSD, Figure 7-left, red-solid line) and also rotation (given by the occurrence of several broader and less-intense peaks in the torsion angle distribution) motions were observed for configuration 2 (where the molecules adopt the most stable conformation found in vacuum), suggesting that the molecules in this conformation are loosely confined and hence are free to move/rotate within the STF cavities. Interestingly, the ${ }^{13} \mathrm{C}$ NMR bands observed for this system were very sharp and well-resolved (Figure 2) (more typically observed in NMR of liquids with high degree of motion of the active nuclear species), what could be explained by this strong motion of the 123TEI cations in this framework; this would suggest that indeed most of the $123 T E$ cations adopt this conformation in the STF cavities, conformation which in fact is the most stable one in vacuum.

\section{Conclusions}

The three imidazolium cations used as SDA in the synthesis of pure silica zeolites produced the STF zeolite, but each cation showed a different specificity for this phase. The performance of the SDAs in directing the crystallization towards the STF zeolite follows the order 123TE4MI > 123TEI > 2E13DMI. On the other hand, only the 123TEI cation (that shows an intermediate hydrophobicity), is also able to produce zeolite MFI when working at considerably high 
$\mathrm{H}_{2} \mathrm{O} / \mathrm{SiO}_{2}$ ratios. The performance of these $123 \mathrm{TEI}$ cations follows the Villaescusa's rule, since the STF zeolite is less dense than MFI; in addition, the STF zeolite is metastable compared to MFI. In order to understand the selectivity of these imidazolium SDAs and explain the experimental observations, a computational study was performed, showing a perfect agreement with the experimental results. In the case of the MFI framework, the order of interaction energies found for this framework was 123TEI > 123TE4MI > 2E13DMI, in agreement with the experimental results; only the 123TEl cation is able to direct the crystallization of this framework because a perfect match of the molecular shape and symmetry and those of the MFI framework is established. Hence, this cation presents a certain template effect in terms of the spatial relationship between the shape of the organic cation and the topology of the MFI structure. Compared to the other two cations, 123TEI shows a greater efficiency in filling the pores of the MFI zeolite, due to their large size that nicely fits with the dimensions of the channel intersections. For the STF zeolite, the order of interaction energies found for this framework, 123TE4MI > 123TEI > 2E13DMI, is also in very good agreement with the experimental results which showed that the three cations allowed the crystallization of this zeolite following the same order of specificity. In this case, the globular-shaped molecules loosely fill the spherical cavities of the STF framework, and the most efficient SDA is the one that more efficiently fills the cavities, i.e. the one with the higher number of $(\mathrm{C}+\mathrm{N})$ atoms (123TE4MI). A much higher degree of motion of the 123TEl cation is observed when occluded within the cavities of the STF framework compared to when hosted in the channel intersections of the MFI framework, possibly explaining the different widths of the ${ }^{13} \mathrm{C} N \mathrm{NR}$ bands observed in the two materials.

\section{Acknowledgements}

Y.M.V acknowledges the CAPES for a master's scholarship. A.R. acknowledges the Consejo Nacional de Ciencia y Tecnología (CONACyT) for a pos-doctoral fellowship. L.G.-H. acknowledges the Spanish Ministry of Economy and Competitivity for a 'Ramón y Cajal' (RYC-2012-11794) contract. Centro Técnico Informático-CSIC is acknowledged for running the calculations. Prof. Dr. H. O. Pastore (UNICAMP) is acknowledged for the Solid State NMR analyses.

\section{Notes and references}

1 a) M. Stöcker, Micropor. Mesopor. Mater., 2005, 82, 257. A. Corma, A. Agustín, Adv. Mater., 1995, 7, 137. b) S. M. Csicsery, J. Catal., 1971, 23, 124.

2 J. D. Sherman, Proc. Natl. Acad. Sci., 1999, 96, 3471.

3 a) M. Comes, M. D. Marcos, R. Martínez-Mañez, M. C Millán, J. V. Ros-Lis, F. Sancenón, J. Soto, L. A. Villaescusa, Chem. Eur. J., 2006, 12, 2162. b) J. S. Sancenón, L. A. Villaescusa, Chem. Eur. J., 2006, 12, 2162. c) M. Comes, G. Rodríguez-López, M. D. Marcos, R. Martínez-Máñez, F. Sancenón, J.Soto, L. A. Villaescusa, P. Amorós, D. Beltrán. Angew. Chem. Int. Edit., 2005, 117, 2978. d) J. V. Ros-Lis, R. Martínez-Máñez, J. Soto, L. A. Villaescusa, K. Rurack, J. Mater. Chem., 2011, 21, 5004.

4 a) G. Calzaferri, M. Pauchard, H. Maas, S. Huber, A. Khatyr, T. Schaafsma, J. Mater. Chem., 2002, $12,1$. b) N. Gfeller, G. Calzaferri, J. Phys. Chem. B., 1997, 101, 1396.

5 a) R. F. Lobo, S. I. Zones, M. E. Davis, J. Incl. Phenom. Mol. Recognit. Chem., 1995, 21, 47. b) J. PérezPariente, L. Gómez-Hortigüela, The Role of Templates in the Synthesis of Zeolites, in Zeolite: From Model Materials to Industrial Catalysts. (eds. J. Čejka, J. Peréz-Pariente, W. J. Roth). ISBN: 978-81-7895-330-4, 2008, 33-62. c) J. Li, A. Corma, J. Yu, Chem. Soc. Rev., 2015, 44, 7112.

6 a) L. A. Villaescusa, M. A. Camblor, Recent Res. Dev. Chem. 1, 2003, 1 ,93. b) P. A. Barrett, T. Boix, M. Puche, D. H. Olson, E. Jordan, H. Koller, M. A. Camblor, Chem. Commun., 2003, 2114. c) M. A. Camblor, L. A. Villaescusa, M. J. Días-Cabañas, Top. Catal., 1999, 9, 59. d) S. I. Zones, S-J. Hwang, S. Elomari, I. Ogino, M. E. Davis, A. W. Burton, C. R. Chimie, 2005, 8, 267.

7 a) R. Simancas, J. L. Jordá, F. Rey, A. Corma, A. Cantín, I. Peral, C. Pospescu, J. Am. Chem. Soc., 2014, 136, 3342. b) J. Jiang, J.L. Jordá, M. J. Díaz-Cabañas, J. Yu, A. Corma, Angew. Chem. Int. Ed., 2010, 49, 4986. c) J. Jiang, J. Yu, A. Corma, Angew. Chem. Int. Ed., 2010, 49, 3130. d) M. J. Díaz-Cabañas, J. Jiang, M. Afeworki, D. L. Dorset, S. L. Soled, K. G. Strohmaiers, Proc. Natl. Acad. Sci. U.S.A., 2010, 107, 13997. e) A. Burton, S. Elomari, R. C. Medrud, I. Y. Chan, C. Y. Chen, L. M. Bull, E. S. Vittoratos, J. Am. Chem. Soc., 2003, 125, 163.

8 J. E. Schmidt, D. Xie, T. Rea, M. E. Davis, Chem. Sci., 2015, 6, 1728.

9 Y. Lorgouilloux, M. Dodin, J-L. Paillaud, P. Caullet, L. Michelin, L. Josien, O. Ersen, N. Bats, J. Solid State Chem., 2009, 182, 622.

10 A. Rojas, M. A. Camblor, Angew. Chem. Int. Ed., 2012, 51, 3854.

11 D. Jo, S. B. Hong, M. A. Camblor, ACS Catal., 2015, 5, 2270.

12 C. W. Lopes, P. H. Finger, M. L. Mignoni, D. J. Emmrich, F. M. T. Mendes, S. Amorim, S. B. C. Pergher, Microporous Mesoporous Mater., 2013, 213, 78.

13 A. Rojas, E. Martínez-Morales, C. M. Zicovich-Wilson, M. A. Camblor, J. Am. Chem. Soc., 2012, 134, 2255.

14 Forcite Module. Material Studio 5.5. Accelrys Inc.

15 P. Dauger-Osguthorpe, V. A. Roberts, D. J. Osguthorpe, J. Wolff, M. Genest, A. T. Hagler, Proteins: Struct. Function Genetics 1988, 4, 21.

16 A. De Vita, M. J. Gillan, J. S. Lin, M. C. Payne, I. Stich, J. L. Clarke, Phys. Rev. B, 1992, 46, 12964.

17 A. Rojas, L. Gómez-Hortigüela, M. A. Camblor, Dalton Trans., 2013, 42, 2562.

18 Conformer Calculation module, Material Studio, version 7.0, Accelrys Inc., San Diego, CA, 2013. 
19 J. E. Schmidt, M. W. Deem, M. E. Davis, Angew. Chem. Int. Ed., 2014. 126, 8512.

20 Y. Kubota, M. M. Helmkamp, S. I. Zones, M. E. Davis, Micropor. Mater., 1996, 6, 213.

21 H. Koller, A Wöker, L. A. Villaescusa, M. J. Díaz-Cabañas, S. Valencia, M. A. CAmblor, J. Am. Chem. Soc., 1999, 121, 3368.

22 L. A. Villaescusa, W. Zhou, R. E. Morris, P. A. Barrett, J. Mater. Chem., 2004, 14, 1982.

23 B. Harbuzaru, M. Roux, J-L. Paillaud, F. Porcher, C. Marichal, J-M. Chézeau, J. Patarin, Chem. Lett., 2002, 616. 